\title{
SECURING VEHICLES
}

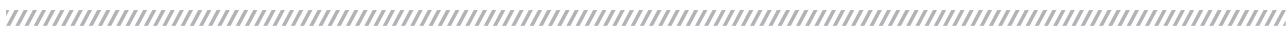

\section{Dear Reader,}

On the last day of March, we had the honour of meeting and interacting with Sachin Lawande, President and CEO, Visteon. We will bring to you a detailed coverage on that meeting in our May edition, but during the hour long interaction, he touched upon something very critical for the times we are living in - the need for cyber security and anti-hacking measures in modern vehicles.

With every added feature on a vehicle today, there are innumerable lines of codes that are being added to the vehicle's on-board system. As the role of software increases multi-fold, microprocessors are taking control over a range of crucial functions including steering, brakes, headlights and cockpit systems. Greater connectivity options in vehicles have also brought greater threats against cyber attacks, and it appears that a full-proof solution is still somewhat far away.

Suppliers have realised the seriousness of this threat, and have lined-up measures to restrict, and eventually nullify such attacks. Even the Society of Automotive Engineers (SAE) has notified a cyber security framework, called J3061, which provides 'needed guidance on ways to design a complex system to perform a desired function, while limiting its ability to be exploited to do something malicious'.

Visteon, on its part, is making a move in this regard. It has decided to set-up a laboratory for white hat hacking in India, in an attempt to find solutions to potential cyber attacks on vehicle systems. Other leading manufacturers also realise the seriousness of this threat, and have taken steps to thwart any such break-ins into vehicles.

Another global supplier, Magna International joined hands with Israeli Argus Cyber Security Ltd in September last year to deliver a robust solution to vehicle security concerns related to cyber attacks. Then, there are other global majors such as HARMAN International and Continental Automotive investing serious amount of effort and energy to find answers.

Experts we've spoken to say there is no single solution yet to stop hackers from getting into a vehicle system. With connectivity increasing in vehicles, and the steady progress being made towards autonomous driving, the security exposures too seem to be increasing. The threat is real, but the good news is, manufacturers are sure of finding solutions, although it may take some time.

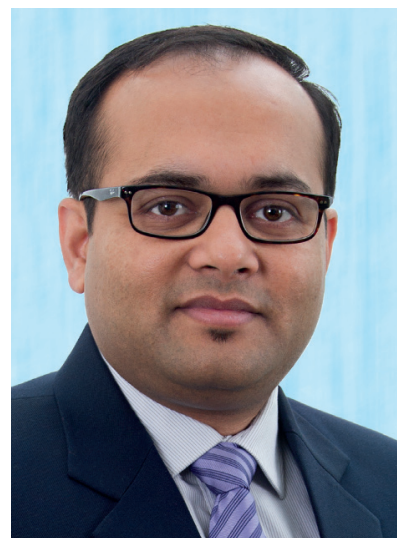

autotechreview.com

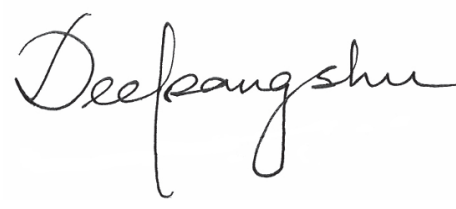

DEEPANGSHU DEV SARMAH

Editor-in-Chief

New Delhi, April 2016 